\title{
Dynamical thermalization in time-dependent Billiards
}

\author{
Matheus Hansen ${ }^{1}$, David Ciro ${ }^{2}$, Iberê L. Caldas ${ }^{1}$ and Edson D. Leonel ${ }^{3}$ \\ 1 Instituto de Física - Universidade de São Paulo, São Paulo - CEP 05508-090 SP, Brazil \\ 2 Instituto de Astronomia Geofísica e Ciências Atmosféricas - \\ Universidade de São Paulo, São Paulo - CEP 05508-090 SP, Brazil \\ 3 Departamento de Física - UNESP, Rio Claro - CEP 13506-900 SP, Brazil
}

(Dated: May 8, 2019)

\begin{abstract}
Numerical experiments of the statistical evolution of an ensemble of non-interacting particles in a time-dependent billiard with inelastic collisions, reveals the existence of three statistical regimes for the evolution of the speeds ensemble, namely, diffusion plateau, normal growth/exponential decay and stagnation. These regimes are linked numerically to the transition from Gauss-like to Boltzmann-like speed distributions. Further, the different evolution regimes are obtained analytically through velocity-space diffusion analysis. From these calculations the asymptotic root mean square of speed, initial plateau, and the growth/decay rates for intermediate number of collisions are determined in terms of the system parameters. The analytical calculations match the numerical experiments and point to a dynamical mechanism for thermalization, where inelastic collisions and a high-dimensional phase space lead to a bounded diffusion in the velocity space towards a stationary distribution function with a kind of reservoir temperature determined by the boundary oscillation amplitude and the restitution coefficient.
\end{abstract}

PACS numbers: 05.45.-a, 05.45.Tp, 05.70.-a

\section{INTRODUCTION}

The Loskutov-Ryabov-Akinshin (LRA) conjecture [1] was proposed as an attempt to foreseen what would happen to the behavior of the average velocity for an ensemble of particles in a time dependent billiard [2, 3] whenever the shape and characteristics of the phase space of the corresponding static version of the billiard was known. Chaos in the phase space for the dynamics of a particle in a billiard with static boundary was claimed by the conjecture to be a sufficient condition to produce unlimited energy growth, also known as Fermi acceleration [4], of the particles when a time perturbation to the boundary was introduced. The conjecture was tested in a number of billiards being therefore validated [ $[5,6]$. A counter example of such conjecture was observed in an elliptic billiard [7, 8], whose structure is integrable in the static form, but that presents an unlimited diffusion of energy when a time-dependence is introduced on the billiard boundary.

The physics behind the unlimited energy growth is understood and is mainly related to the diffusion of velocities as a function of time 9 -12]. Different regimes of growth are related to different shapes of the speed distribution function. The counter-intuitive fact that Hamiltonian dynamics may lead to an unlimited energy growth in chaotic billiards comes from the higher dimension of the dynamical system and such a growth appears to contradict what is expected from thermodynamics. However, this only states that there is not a well defined temperature for the moving boundary, which works here as the energy reservoir, i.e. the wall is not in a thermodynamic equilibrium. In a regular situation, a gas of non-interacting particles with an initial low temperature $T_{0}$ will increase its energy if introduced in a, pre- viously empty, recipient with walls at ambient temperature $T_{a}>T_{0}$. The opposite will happen if the gas is at an initially larger temperature $T_{0}>T_{a}[13$. This thermalization process, in general, manifests as a monotonic change in temperature as time advances, leading to an asymptotic state of thermal equilibrium.

In contrast, consider a conservative chaotic billiard with an oscillating boundary, such that unlimited energy growth is observed. Since the billiard energy is essentially kinetic, the growth of energy leads also to the growth of the temperature. The type of interaction of the particle with the boundary is the reason of such behavior. Elastic collisions preserves both momentum and kinetic energy in the moving referential frame of the boundary, which does not imply conservation of energy for the inertial frame of the gas center of mass, leading to the the unlimited energy growth of the ensemble of particles.

On the other hand, inelastic collisions preserve only momentum, and the dissipation introduced produce drastic topological changes in the phase space. When inelastic collisions are taken into account, the Liouville measure is no longer preserved and attractors can develop in the phase space 14]. Considering that the attractor is located at finite values of the velocity, and its basins of attraction contains most of phase space, it is clear that the individual trajectories will converge to the attractor, and the average speed will saturate leading to a sort of thermodynamical equilibrium for the perturbed billiard.

Until now, important results have been obtained in the characterization of the unbounded energy evolution for particles in chaotic billiards. Our contribution in this context, is the statistical description of the evolution to a final equilibrium, and the close connection of this behavior with thermal equilibrium. To our knowledge this problem has not been addressed elsewhere and offers a significant analogy between dynamical and thermal equi- 
librium.

In this paper we discuss the dynamics of an ensemble of particles moving in an oval billiard with a periodically oscillating boundary. We consider inelastic collisions of the particles with the boundary and explore the behavior of the root mean square of speed considering the shape of the probability distribution function of the speeds. Then we show that the presence of dissipation leads the system towards an asymptotic stationary state, which, with basis on its statistical properties, we argue is a dynamical equivalent of a thermodynamical equilibrium.

The paper is organized as follows. In Sec. III we discuss the equations that compose the billiard model with time-dependent boundary, then in Sec. [II] we show the statistical analysis of the speeds and the diffusion process in the system. In Sec. III we present an analytical derivation of the time evolution of the root mean square of the speeds in terms of the control parameters of the problem. In Sec. IV we offer a connection between the asymptotic dynamics of dissipative time-dependent billiards and the concept of thermalization. Finally, in the last section we present our conclusions and final remarks.

\section{THE TIME-DEPENDENT BILLIARD}

We start considering a time-dependent oval-billiard [15] with boundary described in polar form as

$$
R_{b}(\theta, \epsilon, t, a, p)=1+\epsilon[1+a \cos (t)] \cos (p \theta),
$$

where $R_{b}$ is the boundary radial coordinate, $\theta$ is the polar angle, $\epsilon$ measures the oval deformation, $t$ is the time, $a$ is the boundary oscillation amplitude and $p$ is a positive integer $^{1}$.

The trajectory of a particle inside of the billiard can be described using a nonlinear four-dimensional mapping $H: \mathbb{R}^{4} \rightarrow \mathbb{R}^{4}$, such that $\left(\theta_{n+1}, \alpha_{n+1}, V_{n+1}, t_{n+1}\right)=$ $H\left(\theta_{n}, \alpha_{n}, V_{n}, t_{n}\right)$. The angle $\alpha_{n}$ is measured between the particle trajectory and the tangent line to the boundary at $\left(\theta_{n}, t_{n}\right)$, after the $n^{\text {th }}$ collision with the wall, and $V_{n}=\left|\vec{V}_{n}\right|$ is the velocity magnitude. Figure 1 shows in red a sketch of a typical trajectory of a particle at different times in the model.

Given that there are no additional potentials inside the billiard, each particle moves with constant speed along a straight line between collisions. The radial position of the particle is given by $R_{p}(t)=\sqrt{X_{p}^{2}(t)+Y_{p}^{2}(t)}$, where $X_{p}(t)$ and $Y_{p}(t)$ are the rectangular coordinates at time $t$, which are given by

$$
\begin{aligned}
X_{p}(t) & =X\left(\theta_{n}, t_{n}\right)+\left|\vec{V}_{n}\right| \cos (\mu)\left[t-t_{n}\right], \\
Y_{p}(t) & =Y\left(\theta_{n}, t_{n}\right)+\left|\vec{V}_{n}\right| \sin (\mu)\left[t-t_{n}\right],
\end{aligned}
$$

\footnotetext{
${ }^{1}$ Non integer numbers produce open billiard leading to escape of particle through hole on the border.
}

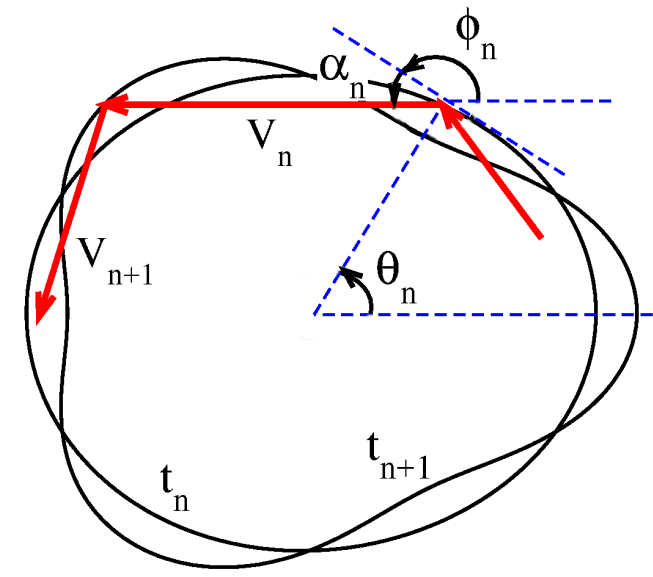

FIG. 1: (Color online). Sketch of two consecutive collisions (red line) of a particle in a time-dependent oval-billiard with $a=0.9, \epsilon=0.08$ and $p=3$.

with $\mu=\left(\alpha_{n}+\phi_{n}\right)$ and $\phi=\arctan \left(Y^{\prime}(\theta, t) / X^{\prime}(\theta, t)\right)$, where $Y^{\prime}(\theta, t)=d Y / d \theta$ and $X^{\prime}(\theta, t)=d X / d \theta$.

The new dynamical variable $\theta$ at collision $n+1$ is obtained through the numerical solution of the implicit equation $R_{b}\left(\theta_{n+1}, t_{n+1}\right)=R_{p}\left(\theta_{n+1}, t_{n+1}\right)$, with the time $t_{n+1}$ given by

$$
t_{n+1}=t_{n}+\frac{\sqrt{\Delta X_{p}^{2}+\Delta Y_{p}^{2}}}{\left|\vec{V}_{n}\right|},
$$

where $\Delta X_{p}=X_{p}\left(\theta_{n+1}, t_{n+1}\right)-X\left(\theta_{n}, t_{n}\right)$ and $\Delta Y_{p}=$ $Y_{p}\left(\theta_{n+1}, t_{n+1}\right)-Y\left(\theta_{n}, t_{n}\right)$.

The reflection laws for each collision of the particle with the boundary can be obtained by applying conservation of momentum in an instantly inertial frame where the contact point of the billiard is at rest. In our case, the reflection laws are

$$
\begin{aligned}
\vec{V}_{n+1}^{\prime} \cdot \vec{T}_{n+1} & =\xi \vec{V}_{n}^{\prime} \cdot \vec{T}_{n+1} \\
\vec{V}_{n+1}^{\prime} \cdot \vec{N}_{n+1} & =-\kappa \vec{V}_{n}^{\prime} \cdot \vec{N}_{n+1}
\end{aligned}
$$

where $\vec{T}_{n+1}=\cos \left(\phi_{n+1}\right) \hat{i}+\sin \left(\phi_{n+1}\right) \hat{j}$ and $\vec{N}_{n+1}=$ $-\sin \left(\phi_{n+1}\right) \hat{i}+\cos \left(\phi_{n+1}\right) \hat{j}$ are the tangent and normal unit vectors, $\vec{V}^{\prime}$ is the particle velocity measured in the non-inertial frame, and $\xi, \kappa \in[0,1]$, are the tangent and normal restitution coefficients respectively.

After collision $n+1$, the tangent and normal components of the velocity are

$$
\begin{aligned}
\vec{V}_{n+1} \cdot \vec{T}_{n+1} & =(1-\xi) \vec{V}_{b} \cdot \vec{T}_{n+1}+\xi \vec{V}_{n} \cdot \vec{T}_{n+1}, \\
\vec{V}_{n+1} \cdot \vec{N}_{n+1} & =(1+\kappa) \vec{V}_{b} \cdot \vec{N}_{n+1}-\kappa \vec{V}_{n} \cdot \vec{N}_{n+1},
\end{aligned}
$$

where

$$
\vec{V}_{b}=\left.\frac{d R_{b}(t)}{d t}\right|_{t_{n+1}}\left[\cos \left(\theta_{n+1}\right) \hat{i}+\sin \left(\theta_{n+1}\right) \hat{j}\right]
$$


is the boundary velocity at time $t_{n+1}$. The magnitude of the particle velocity after collision $n+1$ is

$$
\left|\vec{V}_{n+1}\right|=\sqrt{\left[\vec{V}_{n+1} \cdot \vec{T}_{n+1}\right]^{2}+\left[\vec{V}_{n+1} \cdot \vec{N}_{n+1}\right]^{2}},
$$

and the reflection angle $\alpha_{n+1}$ is

$$
\alpha_{n+1}=\arctan \left[\frac{\vec{V}_{n+1} \cdot \vec{N}_{n+1}}{\vec{V}_{n+1} \cdot \vec{T}_{n+1}}\right] .
$$

\section{SYSTEM EVOLUTION AND SPEED DISTRIBUTION}

In contrast to the static situation, in a time-dependent billiard, particles can gain or lose energy upon interaction with the moving boundary. For an ensemble of particles, the individual gains and losses do not necessarily compensate and the mean energy can change in time. The details of this process can be relevant to understand rates of change in the energy but here we start with a simple heuristic analysis that reveals the broad aspects of the energy evolution.

First of all, consider that the mean quadratic speed changes, by an amount $\psi$ after each collision. We consider here a situation in which there is an small fractional loss of energy after each collision characterized by some restitution coefficient $\gamma<1$, then the mean energy after a collision $n$, satisfies approximately

$$
\overline{V_{n+1}^{2}}=\gamma\left(\overline{V_{n}^{2}}+\psi\right)
$$

This dynamical system has a stable equilibrium when the fractional loss compensates exactly the energy gain after collision. Regardless of the initial configuration, after many collisions, it is expected that the quadratic speed $\overline{V_{n+1}^{2}}$ will approach a stagnation value $V_{\text {sta }}$ given by

$$
V_{\text {sta }}=\sqrt{\frac{\gamma \psi}{1-\gamma}} .
$$

Notice for elastic collisions $\gamma \rightarrow 1$, the stagnation speed diverges, which is consistent with the phenomenon of Fermi acceleration, where there is an unlimited growth of energy as the time evolves [9, 12, 16].

To illustrate numerically the stagnation process we consider the root mean square of the speed distribution for an ensemble of non-interacting particles in the timedependent oval-billiard described in Section I. To reduce numerical fluctuations, in addition to the instantaneous ensemble average, we consider also the time average of the quadratic velocity for the ensemble of particles

$$
V_{r m s}=\sqrt{\frac{1}{M} \sum_{i=1}^{M} \frac{1}{n+1} \sum_{j=0}^{n}|\vec{V}|_{i, j}^{2}},
$$
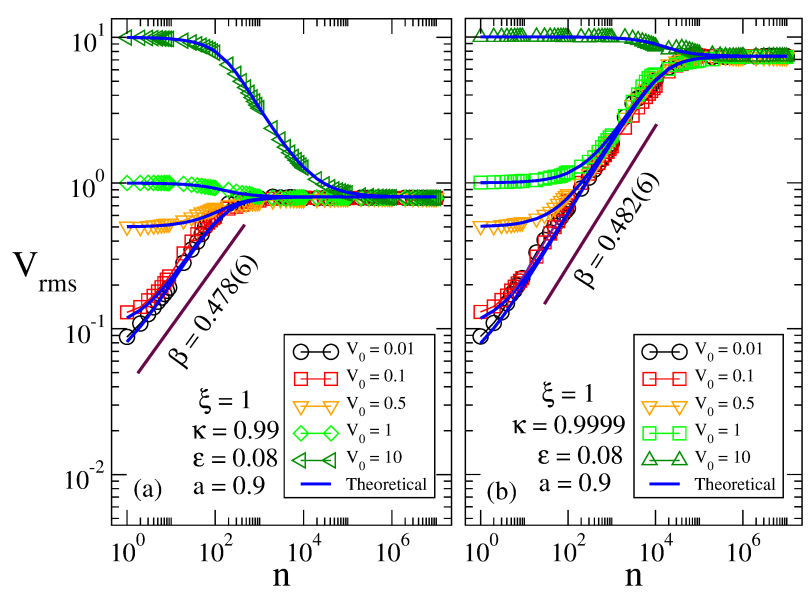

FIG. 2: (Color online). (a,b) Plot for $V_{r m s}$ vs $n$ for different initial speeds and parameters.

where $\vec{V}_{i, j}$ is the velocity of the $i^{\text {th }}$ particle after collision $j$. The first summation is made over an ensemble of different initial conditions randomly chosen in $t \in[0,2 \pi]$, $\alpha \in[0, \pi]$ and $\theta \in[0,2 \pi]$, where all the particles have the same initial speed $V_{0}$, while the second summation is made over the individual orbits. In our simulations we considered an ensemble of $M=10^{6}$ particles colliding $10^{7}$ times with the boundary.

The numerical evolution of $V_{r m s}$ is presented Fig. 2 (a,b) for two different restitution coefficients $\kappa$, and various initial configurations with different $V_{0}$. These curves in Fig. 2 exhibit three different evolution stages for each initial speed. Initially, for speeds around of the maximum speed of the boundary $V_{\max }=a \epsilon, V_{r m s}$ has a plateau, whose extension depends on the initial speed of the particles. After a first crossover, the system enters the growth regime following a power law with exponent $\beta \sim 1 / 2$ of the number of collisions $n$. Finally, a second crossover is observed after which the $V_{r m s}$ saturates at $V_{\text {sta }}$. It can also be observed that when $V_{0} \gg a \epsilon, V_{r m s}$ decays exponentially to the stagnation regime in agreement with the heuristic discussion in Sec. III.

However, to understand in a more detailed fashion the growth rates and transition values we need to take into account the diffusion of particles in the velocity space $\left(V_{x}, V_{y}\right)$. At the initial stage, all particles exist on a circle of radius $V_{0}$. After a collision, each particle jumps by a small amount $\left(\delta V_{x}, \delta V_{y}\right)$ in some direction. Provided there are more available states in the velocity space outside the circle than inside, the probability of particles moving outside the circle is larger than inwards. This small imbalance leads to a growth in the $V_{r m s}$ of the ensemble when the initial radius $V_{0}$ is below $V_{\text {sta }}$. However, the initial growth rate is very small because the initial distribution of particles has to relax towards a Gaussian distribution before exhibiting the usual growth rate for a random walk $\beta_{R W}=1 / 2$. Such relaxation process results in an initial plateau that is longer for larger initial 

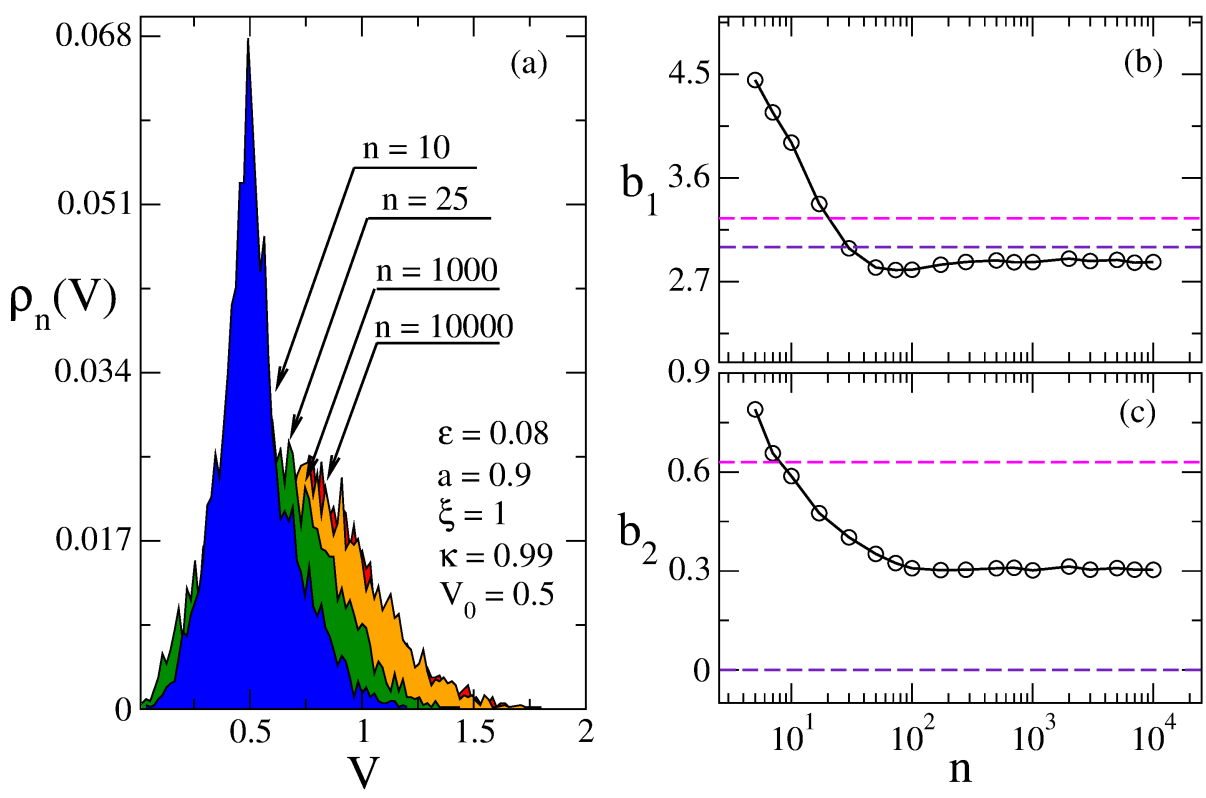

FIG. 3: (Color online). (a) Plot of the evolution of the speed distribution function $\rho_{n}(V)$ for an ensemble of $10^{6}$ particles, with initial speed $V_{0}=0.5$, after different numbers of collisions $n$ and (b,c) plot of the measurement of the kurtosis $b_{1}$ and skewness $b_{2}$ (black curve in both figures) respectively, for the speed distribution function after different number of collisions. The purple and pink dashed lines are the values for the kurtosis and skewness for the Normal and two-dimensional Maxwell-Boltzmann distributions respectively.

speeds. For large initial velocities $V_{0}>V_{\text {sta }}$, the initial plateau occurs in the same fashion, but the probability of moving inwards is larger because with each collision the particles must give up an amount of energy proportional to $V^{2}$. Although there are also losses for small velocities, the diffusion there dominates because the characteristic value of $\delta V^{2}$ is larger than the energy lost after each collision.

In figure 3(a), for the same parameters of Fig. 2(a) and $V_{0}=0.5$, we present the evolution of the speed distribution function $\rho_{n}(V)$ as the number of collisions increases. The $10^{t h}$ collision corresponds to the plateau region of the Fig. 2(a) (for $V_{0}=0.5$ ) where a spreading Gauss-like distribution preserves its mean around the initial speed until it reaches $V=0$ at the left side. At the $25^{\text {th }}$ collision the system is in the growth regime and finally, for the $1000^{\text {th }}$ and $10000^{\text {th }}$ collisions the speed distribution does not change appreciably because the system reached its stagnation regime. Thus, comparing Fig. 2(a) and Fig. 3(a), we can follow, for $V_{0}=0.5$, the $V_{r m s}$ and $\rho_{n}(V)$ evolution as $n$ increases

In order to characterize quantitatively the shape evolution of the speed distribution with the number of collisions, we calculated the kurtosis $b_{1}$ and skewness $b_{2}$ for $\rho_{n}(V)$ as functions of the number of collisions with usual definitions [17], given by

$$
b_{1}=\frac{1}{M} \sum_{i=1}^{M}\left[\frac{V_{i}-\bar{V}}{\sigma}\right]^{4},
$$

$$
b_{2}=\frac{1}{M} \sum_{i=1}^{M}\left[\frac{V_{i}-\bar{V}}{\sigma}\right]^{3}
$$

where $\sigma_{V}=\sqrt{\left\langle V^{2}\right\rangle-\langle V\rangle^{2}}$.

Figure 3(b,c) shows the evolution of $b_{1}$ and $b_{2}$ for the speed distribution function presented in Fig. 3(a). This figure also shows the values of $b_{1}$ and $b_{2}$ for the Normal (purple dashed line) and a two-dimensional MaxwellBoltzmann (pink dashed line) distributions, which differ from ours because of the stochastic nature of their associated processes.

Notice that after $\rho_{n}(V)$ reaches the stagnation regime (about 100 collisions), the kurtosis measurement $\left(b_{1} \approx\right.$ $2.87)$ is close to that of a Normal distribution, while the skewness measurement $\left(b_{2} \approx 0.30\right)$ is an intermediary value between the Normal and the Maxwell-Boltzmann distributions.

\section{KINETIC ANALYSIS}

Consider that the quadratic speed of a single particle, $\tilde{V}^{2}$, changes by an amount $\psi(\alpha, \theta, t, V)$ after colliding with the boundary at position $\theta$ with incidence angle $\alpha$ at time $t$,

$$
\tilde{V}^{2}(\alpha, \theta, t, V)=V^{2}+\psi(\alpha, \theta, t, V),
$$

where $V^{2}$ and $\tilde{V}^{2}$ are the quadratic speeds before and after collision. In an ensemble of $M$ particles, there are 
approximately $M_{\mathcal{F}_{n}}(\alpha, \theta, t, V) d \alpha d \theta d t d V$ particles with variable $x$ between $x$ and $x+d x$, where $x=\{\alpha, \theta, V, t\}$. Then, it is possible to describe the mean quadratic speed after the $n^{\text {th }}$ collision of an ensemble of particles as

$$
\overline{V_{n+1}^{2}}=\overline{V_{n}^{2}}+\delta \overline{V_{n}^{2}}
$$

where

$$
\begin{aligned}
\overline{V_{n}^{2}}=\int_{0}^{\infty} \int_{0}^{2 \pi} \int_{0}^{2 \pi} \int_{0}^{\pi} & V^{2} \mathcal{F}_{n}(\alpha, \theta, t, V) d \alpha d \theta d t d V, \\
\delta \overline{V_{n}^{2}}=\int_{0}^{\infty} \int_{0}^{2 \pi} \int_{0}^{2 \pi} \int_{0}^{\pi} & \psi(\alpha, \theta, t, V) \mathcal{F}_{n}(\alpha, \theta, t, V) \times \\
& d \alpha d \theta d t d V,
\end{aligned}
$$

and $\mathcal{F}_{n}(\alpha, \theta, t, V)$ is the full phase space distribution function after collision $n$. In our case, we can factor this distribution as

$$
\mathcal{F}_{n}(\alpha, \theta, t, V)=F(\theta, \alpha) \rho_{V}(t) \rho_{n}(V),
$$

where $F(\alpha, \theta)$ are the angles distribution, $\rho_{V}(t)$ is the collision time distribution and $\rho_{n}(V)$ is the speed distribution function.

As observed in Fig. $4(\mathrm{a}-\mathrm{f})$, the angles $F(\alpha, \theta)$ and collision time distributions $\rho_{V}(t)$, are almost unaffected by the amplitude of the boundary oscillations $a$. Consequently they are almost independent of the index $n$ and on the initial speed values. This can be understood in terms of the phase-space projection $\theta-\alpha$, that retains important features of the unperturbed problem, which is independent on the velocity of the particles and contains large regular regions with invariant torii that modulate the size of the chaotic region as a function of the angles $\theta-\alpha$, in a way consistent with Fig. 4 .

On the other hand, as discussed in the previous section, the speed distribution function $\rho_{n}(V)$ (see Fig. B(a)) depends on both the speed and the index $n$.

Using decomposition (19), we want to find an analytical expression that describes the behavior of the root mean square speed shown in Fig. 2(a,b). As discussed in [12], to determine the behavior of the mean quadratic speed it is not necessary to describe the evolution of the global distribution function, but only to know the evolution of its first momenta. Therefore, replacing the Eq. (19) in Eq. (17), and defining the partial mean

$$
W(V)=\int_{0}^{2 \pi} \int_{0}^{2 \pi} \int_{0}^{\pi} \psi(\alpha, \theta, t, V) F(\theta, \alpha) \rho_{V}(t) d \alpha d \theta d t,
$$

we can write a more compact expression for the change of the mean quadratic speed as

$$
\delta \overline{V_{n}^{2}}=\int_{0}^{\infty} \rho_{n}(V) W(V) d V .
$$

As detailed in [12], if we make a second-order expansion of the $W(V)$ around the mean speed $\overline{V_{n}}$ of the speed distribution function $\rho_{n}(V)$, we obtain

$$
W(V) \approx W\left(\overline{V_{n}}\right)+W^{\prime}\left(\overline{V_{n}}\right)\left(V-\overline{V_{n}}\right)+\frac{1}{2} W^{\prime \prime}\left(\overline{V_{n}}\right)\left(V-\overline{V_{n}^{2}}\right) .
$$

Inserting the Eq. (22) in Eq. (21), we obtain an approximation for the change of the mean quadratic speed using the second-order expansion, as follows

$$
\delta \overline{V_{n}^{2}}=W\left(\overline{V_{n}}\right)+\frac{1}{2} W^{\prime \prime}\left(\overline{V_{n}}\right)\left(\bar{V}-\overline{V_{n}^{2}}\right) .
$$

Notice also the Eq. (22) is accurate only around the distribution mean. As we get far from the mean value the approximation becomes poorer. However, $\rho_{n}(V)$ in the integrand of the Eq. (21) drops for large and small values of the speed, so the integrand is small where the expansion of $W(V)$ is not accurate. The interested reader can find more details about these approximations in 12 .

Finally, replacing the Eq. (23) in Eq. (16), we find a second-order approximation for the mean quadratic speed after collision $n$, as follows

$$
\overline{V_{n+1}^{2}}=\overline{V_{n}^{2}}+W\left(\overline{V_{n}}\right)+\frac{1}{2} W^{\prime \prime}\left(\overline{V_{n}}\left(\overline{V_{n}^{2}}-\overline{V_{n}^{2}}\right) .\right.
$$

In order to determine the partial mean $W(V)$, we first need to find $\psi(\alpha, \theta, t)$, which depends on the particular problem. In this case, the equations of the timedependent oval-billiard lead us to

$$
\begin{aligned}
\psi(\alpha, \theta, t)= & \left(\kappa^{2}-1\right) V^{2} \sin ^{2}(\alpha)+ \\
& +(1+\kappa)^{2}(a \epsilon)^{2} \cos ^{2}(p \theta) \sin ^{2}(t)+ \\
& +2 V \kappa a \epsilon(1+\kappa) \sin (\alpha) \cos (p \theta) \sin (t) .
\end{aligned}
$$

Assuming that the collision time distribution $\rho_{V}(t)$ is approximately uniform, i.e

$$
\rho_{V}(t)=\frac{1}{2 \pi},
$$

and replacing the Eq. (25) and Eq. (26) in Eq. (20), we obtain

$$
W(V)=\eta_{1}\left(\kappa^{2}-1\right) V^{2}+\frac{1}{2}(1+\kappa)^{2}(a \epsilon)^{2} \eta_{2},
$$

where

$$
\begin{aligned}
& \eta_{1}=\int_{0}^{\pi} \sin ^{2}(\alpha) F(\alpha, \theta) d \theta d \alpha, \\
& \eta_{2}=\int_{0}^{2 \pi} \cos ^{2}(p \theta) F(\alpha, \theta) d \theta d \alpha,
\end{aligned}
$$

which after inserted in the Eq. (24) result in

$$
\overline{V_{n+1}^{2}}-\overline{V_{n}^{2}}=\eta_{1}\left(\kappa^{2}-1\right) \overline{V_{n}^{2}}+\frac{1}{2}(1+\kappa)^{2}(a \epsilon)^{2} \eta_{2} .
$$

Considering the approximation of continuous limit $\overline{G_{n+1}}-\overline{G_{n}} \approx d G(n) / d n$, we found a solution for the mean quadratic speed

$$
\overline{V^{2}}=\Psi+\left(V_{0}^{2}-\Psi\right) e^{\eta_{1}\left(\kappa^{2}-1\right) n},
$$

where

$$
\Psi=\frac{(a \epsilon)^{2}}{2} \frac{\eta_{2}}{\eta_{1}}\left(\frac{1+\kappa}{1-\kappa}\right) .
$$



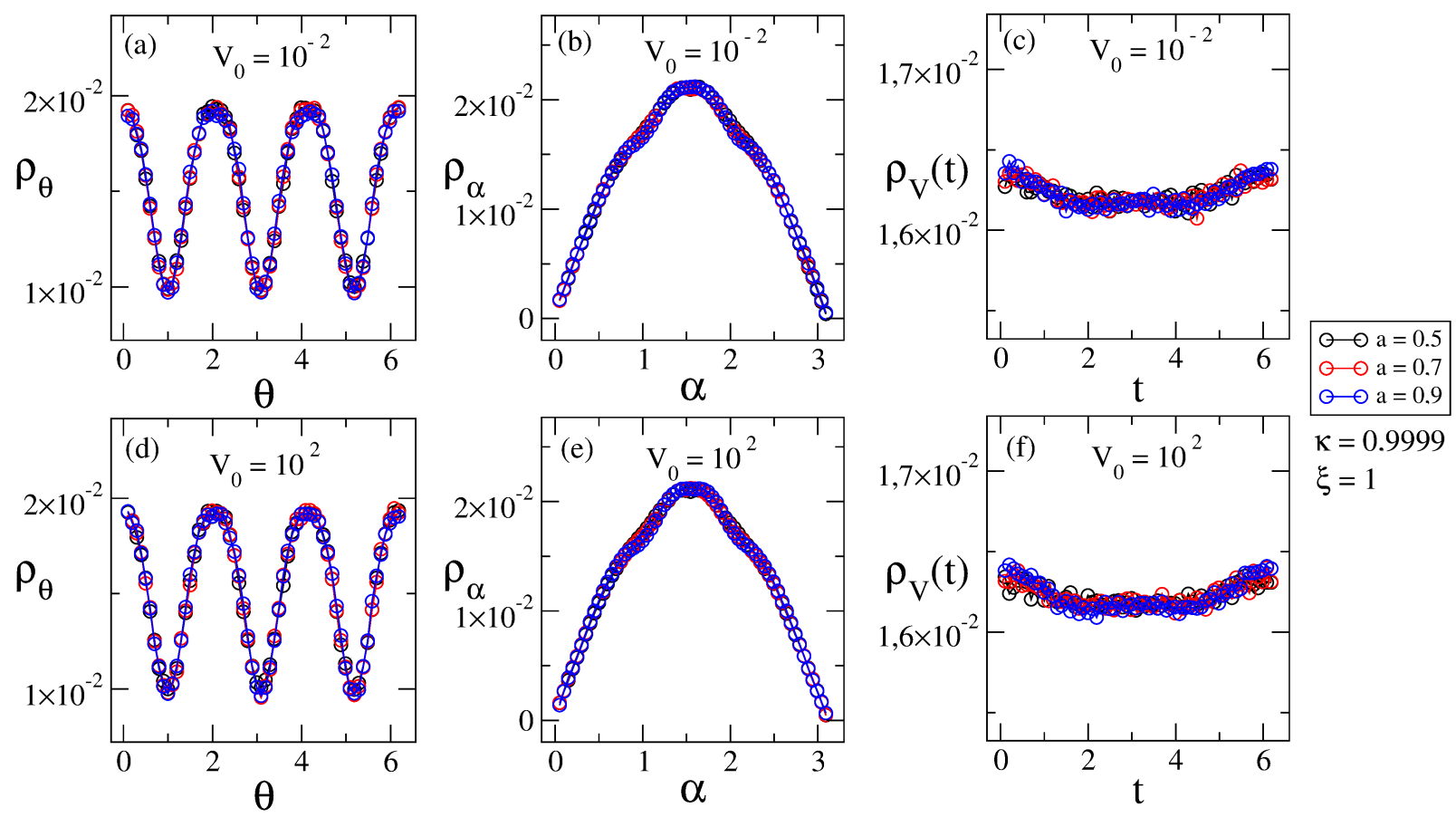

FIG. 4: (Color online). (a,b,d,e) Plot of the numerical distribution functions $\rho_{\theta}(\theta)=\int \tilde{F}(\theta, \alpha) d \alpha$ and $\rho_{\alpha}(\alpha)=\int \tilde{F}(\theta, \alpha) d \theta$, while $(\mathrm{c}, \mathrm{d})$ plot of the numerical collision time distribution functions $\rho_{V}(t)$ for the time-dependent oval-billiard at various amplitude of oscillations and two initial speeds. The control parameters are $\epsilon=0.08$ and $p=3$.

To compare with the previous numerical simulations we need to average over the ensemble of velocities and the history of velocities of all particles. Then we need to average the previous instantaneous mean along the history of all quadratic means. i.e

$$
\overline{V^{2}}=\frac{1}{n+1} \sum_{i=0}^{n} \overline{V_{i}^{2}}
$$

Provided that the arguments of the exponential are negative, their summation converges to

$$
\sum_{i=0}^{n} e^{\eta_{1}\left(\kappa^{2}-1\right) i}=\left[\frac{1-e^{(n+1) \eta_{1}\left(\kappa^{2}-1\right)}}{1-e^{\eta_{1}\left(\kappa^{2}-1\right)}}\right] .
$$

Replacing the Eq. (33) in Eq. (31), we obtain

$$
\overline{V^{2}}=\Psi+\left(\frac{V_{0}^{2}-\Psi}{n+1}\right)\left[\frac{1-e^{(n+1) \eta_{1}\left(\kappa^{2}-1\right)}}{1-e^{\eta_{1}\left(\kappa^{2}-1\right)}}\right] .
$$

The final expression that describes the root mean square speed evolution is

$$
V_{r m s}=\sqrt{\Psi+\left(\frac{V_{0}^{2}-\Psi}{n+1}\right)\left[\frac{1-e^{(n+1) \eta_{1}\left(\kappa^{2}-1\right)}}{1-e^{\eta_{1}\left(\kappa^{2}-1\right)}}\right]},
$$

which corresponds to the continuous line (blue) in Fig. (2) (a,b) in excellent agreement with the numerical results for the analyzed cases.
To conclude this analytical approach, we consider a few relevant limit cases for the Eq. (35) that give us relevant insight on the overall behavior of the obtained solution. When $n=0$, we have

$$
V_{r m s}=V_{0},
$$

and for $n \rightarrow \infty$ we obtain the finite stagnation value

$$
V_{r m s}=a \epsilon \sqrt{\frac{1}{2} \frac{\eta_{2}}{\eta_{1}}\left(\frac{1+\kappa}{1-\kappa}\right)} .
$$

Finally, we consider the intermediate values of $n$ for small initial speeds $V_{0}<<\sqrt{\Psi}$. In the limit of $\kappa \approx 1$ we can expand to a first order the exponential denominator in the Eq. (35), while the numerator is taken to the second order due to the factor $n+1$ that contributes further to its nonlinearity. After a short algebra we obtain

$$
V_{r m s} \cong a \epsilon \sqrt{\frac{\eta_{2}}{2}(1+\kappa)(n+1)},
$$

which for $n \gg 1$ can be approximated to

$$
V_{r m s} \cong a \epsilon \sqrt{\frac{\eta_{2}}{2}(1+\kappa)} n^{1 / 2} .
$$

which leads to the observed growth rate in the numerical treatment of the system, and also depends on the boundary oscillation amplitude, the angles distribution contained in $\eta_{2}$, and on the restitution coefficient $\kappa$. 


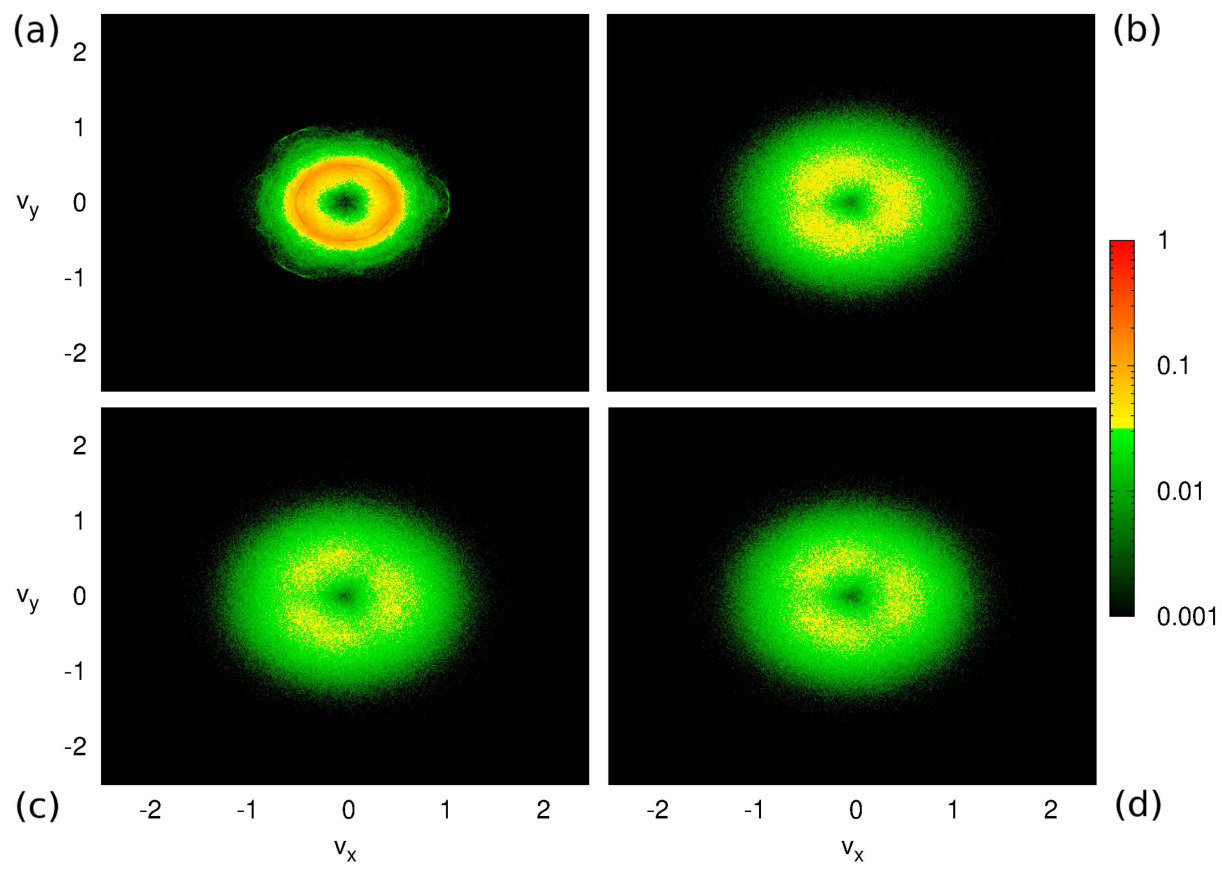

FIG. 5: (Color online). Plot of the diffusion speed in the velocities space after (a) 10 collisions; (b) 25 collisions; 7c) 1000 collisions and (d) 10000 collisions for an ensemble of $10^{6}$ particles with random positions and locations but with the same initial speed $V_{0}=0.5$. The logarithmic scale of colors represents the density of velocity in the velocities space, where the most dense regions are shown in red, while the less are in black.

\section{CONNECTION WITH THERMODYNAMICS}

As a final remark, we present an analogy between dynamical diffusion and the stagnation of the mean quadratic speed with the concept of thermalization. We start by borrowing the concept of temperature in a gas of non-interacting particles inside of a closed region as being proportional to the mean of the quadratic peculiar velocities, which, in our case is simply the mean of the quadratic velocities because the mean velocity of the gas is zero. In this sense, high temperatures are linked with high speeds, while the opposite is also true [18].

We consider the diffusion process in the velocity space $\left(V_{x}, V_{y}\right)$ to understand how the ensemble modify with collision. In order to characterize such diffusion, we evolve an ensemble of $10^{6}$ particles in the velocity space, where each one started with the same velocity at some point in a circle with radius $V_{0}=0.5$ and random angular position in the billiard.

Figure $5(\mathrm{a}-\mathrm{d})$ shows the $\left(V_{x}, V_{y}\right)$ space after 10, 25, 1000 and 10000 collisions, respectively. The color scale measures the density of particles. Note that, after $10 \mathrm{col}-$ lisions (see Fig. 5(a)) the velocities become distributed around the original circle of radius $V_{0}=0.5$, with concentrated the higher density until the particles start populating the velocities near zero. This behavior is in agreement with the previous discussion of the initial plateau discussed in the previous section (see Fig. 2(a). After 25 collisions the velocities become more spread, and the high concentration circle increases its radius, in agreement with the mean speed growth observed when the speed distribution becomes asymmetrical. Finally, after 1000 and 10000 collisions, the distribution does not change much, which, expectedly, corresponds to the stagnation state, for which the individual velocity fluctuations do not affect the distribution function (Fig. 3(a)).

It is interesting to notice that, the velocity fluctuations are responsible to change the mean value of the ensemble of velocities in the space $\left(V_{x}, V_{y}\right)$, where these fluctuations might be estimated as the measure of the variance

$$
\sigma_{V}^{2}=\left\langle\vec{v}^{2}\right\rangle-\langle\vec{v}\rangle^{2}
$$

where the mean velocity $\langle\vec{v}\rangle$ is zero because the particles are inside of a non-translating closed billiard, while the same does not apply for $\left\langle\vec{v}^{2}\right\rangle$, which can be identified as the Eq. (31).

Given that we know how is the diffusion process in the system, we can define an analogous quantity to the temperature named dynamical temperature $T_{d}$, that takes into account the characteristics of dynamical system studied. This quantity can be written as

$$
T_{d} \propto \overline{V^{2}},
$$

where the equality comes after the introduction of a suit- 


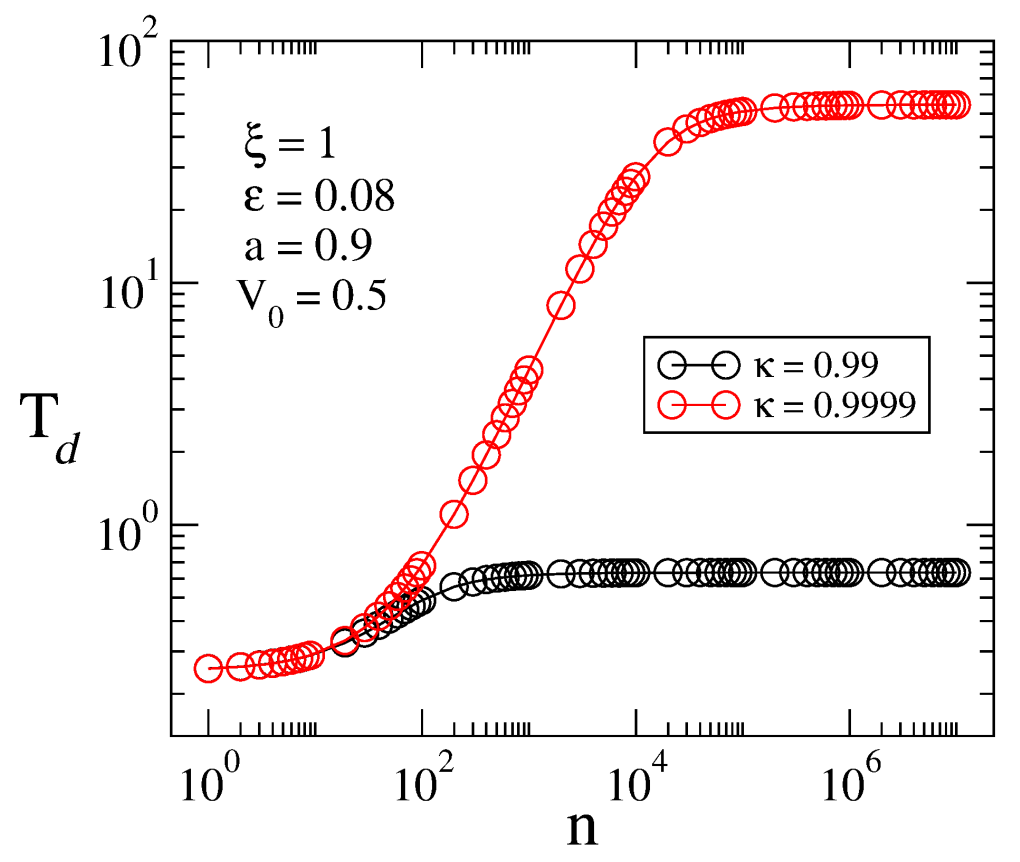

FIG. 6: (Color online). Plot of evolution of the dynamical temperature for a gas of non-interacting particles in a time-dependent oval billiard in function of the number of collisions.

able constant $K_{d}$, which leads us to

$$
T_{d}=\frac{m}{2 K_{d}}\left[\Psi+\left(V_{0}^{2}-\Psi\right) e^{\eta_{1}\left(\kappa^{2}-1\right) n}\right]
$$

with $m$ being the mass of each particle, and $K_{d}$ plays the role of the Boltzmann constant in our dynamical ensemble.

Figure 6 shows the numerical evolution of the dynamical temperature $T_{d}$ as a function of the number of collisions $n$, for two restitution parameters close to one. As can be seen, when the initial speed $V_{0}$ is less than $\Psi$, the dynamical temperature of the gas increases with the collisions until it reaches the stagnation regime, where it remains for the rest of the simulation. The stagnation regime in our context is analogous to a system thermalization with a heat reservoir at constant temperature $T_{\text {sta }}$ which emerges from the interplay between the oscillating boundary and the inelastic collisions.

Provided there are no additional potentials acting inside of the billiard the particles energy is purely kinetic $U_{\text {tot }}=E_{k}$, so that, we can use the dynamical temperature to recast the total energy in terms of it

$$
\begin{aligned}
U_{t o t} & =E_{k}, \\
U_{t o t} & =\frac{1}{2} N m \overline{V^{2}}, \\
U_{t h} & =N K_{d} T_{d},
\end{aligned}
$$

where $N$ is the number of particles. Expectedly, due to the definition of dynamical temperature, we recover an energy equation analogous to an ideal gas at temperature $T_{d}$.

\section{CONCLUSIONS}

In this work we have described both numerically and analytically the development of different evolution regimes for the root mean square of speed an ensemble of non-interacting particles in an oscillating billiard with collision looses. The analytical treatment was based in the diffusion process in the velocity space and resulted in a consistent description for all the regimes of the system evolution with considerable accuracy. The velocity plateau occurs due to the relaxation of the initial configuration, then, for small initial velocities, the energy grows with a characteristic exponent close to $1 / 2$, characteristic of normal diffusion otherwise, it will decrease exponentially for velocities larger than the saturation speed. Finally, the ensemble reaches an stagnation state independent of the initial configuration.

The stagnation regime is analogous to a thermalized state, where the distribution function becomes stationary and its temperature can be used to characterize the energy reservoir, here encompassing the vibrating boundary and the restitution constant. A dynamical temperature was defined to make a connection with thermodynamics that resulted in analogous equations for the energy of an ideal gas in an actual thermal system.

\section{ACKNOWLEDGEMENTS}

$\mathrm{MH}$ and DC thanks to CAPES for financial support. ILC and EDL thanks to CNPq (300632/2010-0 
and 303707/2015-1) and São Paulo Research Foundation (FAPESP) (2018/03211-6 and 2017/14414-2) for their fi- nancial support.

(2008).

[11] V. Gelfreich, V. Rom-Kedar and D. Turaev, Chaos, 22, 033116 (2012).

[12] M. Hansen, D. Ciro, I. L. Caldas and E. D. Leonel, EPL, 121, 60003 (2018).

[13] E. D. Leonel, M. V. C. Gália, L. A. Barreiro and D. F. M. Oliveira, Phys. Rev. E, 94, 062211 (2016).

[14] H. Goldstein, C. P. Poole and J. L. Safko, Classical Mechanics, Pearson, (2002).

[15] M. V. Berry, Eur. J. Phys., 2, 91 (1981).

[16] A. J. Lichtenberg, M. A. Lieberman and R. H. Cohen, Physica D: Nonlinear Phenomena, 1, 291 (1980).

[17] A. Spanos, Probability Theory and Statistical Inference, New York: Cambridge University Press, (1999).

[18] F. Reif, Fundamentals of Statistical and Thermal Physics, Waveland Press, (2009).

[10] V. Gelfreich and D. Turaev, J. Phys. A, 41, 212003 